\title{
Luminol-dependent chemiluminescence by hemocytes of two marine bivalves, Ostrea edulis and Crassostrea gigas
}

\author{
E. Bachère, D. Hervio, E. Mialhe \\ IFREMER, Laboratoire de Biologie et Ecologie des Invertébrés Marins, Unité de Recherche en Pathologie, Immunologie et \\ Génétique Moléculaire, BP 133, F-17390 La Tremblade, France
}

\begin{abstract}
Microbicidal phagocytic function and oxidative metabolism of the hemocytes of 2 oyster species, Ostrea edulis and Crassostrea gigas, were investigated using a luminol-enhanced chemiluminescence (CL) technique. First, experimental parameters adapted to marine bivalve hemocytes were established on hemolymph pools of $C$. gigas in order to obtain qualitatively and quantitatively homogeneous samples and so to perform statistically comparable assays. The use of Modified Alsever Solution (MAS) allowed hemocytes to be kept non-aggregated and in a non-stimulated state until starting the assays. A number of aliquots of $2 \times 10^{5}$ hemocytes with an MAS final concentration of $3.5 \%$ showed high chemiluminescent response after stimulation by zymosan particles. A particle:hemocyte ratio of 80:1 gave optimal CL activity. Activity was inhibited by cytochalasin B, a phagocytic inhibitor, and by sodium azide, the latter indicating the involvement of oxygen products. Having thus defined suitable parameter values, the CL protocol was then applied to $O$. edulis for qualitative and quantitative analyses of respiratory burst capacity at the species and individual levels. Results suggested inter- and intra-specific variability of CL responses. Under the same experimental conditions, $O$. edulis hemocytes generally displayed higher CL activities than C. gigas. Moreover, clear individual variability was demonstrated. The same experimental number of hemocytes showed great differences in CL responses between individuals suggesting a possible correlation with hemogram characteristics such as cell type percentages.
\end{abstract}

\section{INTRODUCTION}

The phagocytic functions of mollusc hemocytes have been widely investigated by optical and electron microscopy, generally using inert material or bacteria as stimulating agents (Bang 1975), and more recently native pathogens because of the availability of specific isolation and purification protocols (Mialhe et al. 1988). These observations led us to compare mollusc hemocytes with immune cells of better-studied animal species in which phagocytosis constitutes an important way of control and elimination of a wide range of pathogens, microorganisms and tumor cells. In vertebrates, phagocytic processes are linked to subsequent microbicidal activities which rely on an oxygen-independent system (Spitznagel 1984) and on oxidative metabolism (Andrew et al. 1985, Thomas et al. 1988). Oxidative metabolic events, known as respiratory burst, are characterized by the production of active oxygen species (Badwey \& Karnosky 1980, Halliwell \& Guteridge 1984). These powerful oxidants are effective as tumoricidal and antimicrobial agents and react with the internalized particles in various processes associated with oxidation-reduction reactions and the generation of photons. This last phenomenon of light emission, which can be detected in chemiluminescence (CL), is frequently used for studying phagocytosis and cell-mediated cytotoxicity in known phagocytes such as neutrophils, eosinophils and mononuclear cells (Allen et al. 1972, Trush et al. 1978).

CL may be regarded as having acquired the status of reference methodology in immunology, since it provides an in vitro measure of the immune state of an animal (Fromtling \& Abruzzo 1985). The existence of this methodology led to the application of CL to invertebrates, notably to several medically important gastropod species, in order to assess their immune response against transmitted parasites such as Schistosoma (Dikkeboom et al. 1988a). Thus, the ubiquitous feature of respiratory burst was discovered in molluscs. In the scallop Patinopecten yessoensis (Bivalvia) respiratory burst phenomena were demonstrated by a histochemical method (Nakamura et al. 1985).

This paper describes the establishment of experi- 
mental parameters suitable for luminol-enhanced CL assays on hemocytes of the marine bivalve Crassostrea gigas. CL was applied to qualitative and quantitative analyses of the respiratory burst in order to validate the hemocyte phagocytic response as a potential monitor of the oyster defense capacity. The standardization of the method permitted study of individual CL responses in 2 bivalve species, C. gigas and Ostrea edulis. Moreover, following the example of vertebrates, for which blood cell types exhibit different functions, a correlation was sought between CL activities and hemogram characteristics as an aid to understanding the variability of oyster individual response.

\section{MATERIALS AND METHODS}

Animals. Three-year-old flat oysters Ostrea edulis were collected from the Etang de Thau, France, (Mediterranean Sea) which is an area free of parasites Bonamia ostreae. Three-year-old Japanese oysters Crassostrea gigas originated from Marennes-Oleron, France (Atlantic Ocean).

Hemolymph sampling. Hemolymph samples were collected from the pericardial cavity and, generally, simultaneously 2-fold diluted with anti-aggregant Modified Alsever Solution (MAS) (Bachère et al. 1988). The hemolymph samples were used either separately or pooled (10 individual hemolymphs).

Hemogram determination. Hemocyte counts were performed, using a Malassez hemacytometer, on hemolymph samples diluted in MAS as just described. For the estimation of numbers of different hemocyte types, a second blood withdrawal from the same individual without MAS was necessary to avoid disruption of slide-adherent hemocytes by the MAS. The pure hemolymph samples $(50 \mu \mathrm{l})$ were deposited on microscope slides and $50 \mu$ of filtered seawater (FSW) was added. The hemocytes were allowed to settle for 30 min at room temperature in a moist chamber. Then, the hemocytes were identified by vital staining using the acridine orange ethidium bromide method (Herzenberg 1979). Briefly, $100 \mu \mathrm{l}$ of the test mixture in FSW was added to the settled hemocytes, which were then observed with an epifluorescence microscope. After counting 200 hemocytes, the percentages of granulocytes and small and large hyalinocytes were estimated.

Zymosan. Zymosan particles (Sigma, USA) were used as a phagocytic stimulus. They were suspended (40 $\mathrm{mg} \mathrm{ml}^{-1}$ ) in $\mathrm{FSW}$, heated for $30 \mathrm{~min}$ at $100^{\circ} \mathrm{C}$, washed and finally suspended in FSW, aliquoted and stored at $-20^{\circ} \mathrm{C}$. At the time of use, the exact number of zymosan particles, in a thawed aliquot, was estimated using a Malassez hemacytometer

Phagocytosis assays. Phagocytosis assays were per- formed by adding $100 \mu$ l of a zymosan suspension to adherent hemocytes on microscope slides, prepared from pure hemolymph samples. After about 30 min incubation, the preparations were washed with FSW, fixed with methanol and finally stained with Hemacolor Kit (Merck). Similar assays were made with hemocyte preparations from MAS-diluted hemolymph samples, washed in advance and resuspended in FSW. In each preparation, the phagocytic activity of the hemocytes was studied by light microscopy.

Chemiluminescence assays. General protocol: Generation of $\mathrm{CL}$ was measured with a liquid scintillation counter (Tri-Carb 2200 CA, Packard) in the out-ofcoincidence mode. The counter was set on repeated cycles and sequential 0.5 min counting periods at fixed intervals, generally for $5 \mathrm{~h}$. The assays were performed at room temperature. Each plastic scintillation vial contained $2 \times 10^{5}$ hemocytes obtained by diluting an adequate quantity of a hemolymph/MAS (1:1) sample with FSW. Luminol (3-aminophtalhydrazide, Sigma, USA) solutions were prepared just before use from a stock solution [10-1 $\mathrm{M}$ in DMSO (dimethyl sulfoxide) Sigma] and $200 \mu \mathrm{l}$ was introduced into each vial, in a total volume of $2 \mathrm{ml}$. The CL base activity of the hemocytes was recorded for a first run. Then, the zymosan suspension was added at a selected zymosan particle-to-hemocyte ratio and the $\mathrm{CL}$ responses were measured. Generally, a vial was kept without stimulus as a control for the measurement of CL base activity during the experiments.

Experimental parameters: The experimental parameters were established with Crassostrea gigas hemocytes.

Effect of MAS concentration. Hemolymph samples were obtained as previously described but diluted 2fold in FSW instead of MAS. The CL assay was then performed, according to the general protocol, with $2 \times$ $10^{5}$ hemocytes, stimulated by zymosan (with a particle:hemocyte ratio of $80: 1)$ and with luminol at a final concentration of $10^{-4} \mathrm{M}$. CL measurements were recorded for these stimulated hemocytes in parallel with non-stimulated ones.

The hemolymph sample withdrawn in MAS from one oyster $\left(2 \times 10^{6}\right.$ hemocytes $\left.\mathrm{mI}^{-1}\right)$ was distributed at 100 $\mu l\left(2 \times 10^{5}\right.$ hemocytes $)$ per vial. Then, appropriate quantities of MAS and FSW were added in order to obtain, in a $2 \mathrm{ml}$ final volume, an MAS concentration series corresponding to $2.5,3.5,4.5,5.5,6.5,7.5,8.5,9.5$ and $10.5 \%$. For the assay, the final concentration of luminol was $10^{-4} \mathrm{M}$, and the hemocytes were stimulated by zymosan at a ratio of $80: 1$.

Effect of luminol concentration. From a $10^{-1} \mathrm{M}$ luminol stock solution, a concentration series was prepared in FSW in order to obtain luminol concentrations of $10^{-3}, 10^{-4}, 10^{-5}, 10^{-6}$ and $0 \mathrm{M}$ luminol. Hemocyte/ 
MAS samples $\left(2 \times 10^{5}\right.$ hemocytes vial $\left.{ }^{-1}, 2.5 \% \mathrm{MAS}\right)$ all obtained from pooled hemolymph from 10 oysters were stimulated by zymosan at a ratio of 80:1.

Effect of zymosan stimulus dose. The CL responses of the hemocytes of pooled hemolymph from 10 individuals were measured after stimulation at different zymosan particle:hemocyte ratios, viz. 20:1, 40:1, 60:1, 80:1, 100:1, 120:1, 140:1, 160:1. The luminol concentration was $10^{-4} \mathrm{M}$.

Inhibition assays with Crassostrea gigas hemocytes: The inhibitory effect of cytochalasin B (Sigma; $1.5 \mu \mathrm{g}$ $\mathrm{ml}^{-1}$ ) was determined by adding $3 \mu \mathrm{l}$ of stock solution (1 $\mathrm{mg} \mathrm{ml}^{-1}$ in DMSO) to the hemocyte suspensions just before starting the phagocytic stimulation. The effect of sodium azide $\left(\mathrm{NaN}_{3}\right.$, Sigma; $\left.32 \mu \mathrm{g} \mathrm{ml}^{-1}\right)$ was determined by adding $5 \mu \mathrm{l}$ of stock solution when the CL responses reached the peak activity. These experiments were performed according to the general protocol: $2 \times 10^{5}$ hemocytes vial ${ }^{-1}$ obtained from pooled samples of hemolymph/MAS; MAS $3 \%$ final concentration; luminol $10^{-4} \mathrm{M}_{\text {; }}$ zymosan particle:hemocyte ratio of $80: 1$.

CL assays of individual hemolymph samples: Hemolymph samples were individually withdrawn, simultaneously diluted 2-fold in MAS, from 10 oysters of each of the 2 species. The CL assays were performed according to the general protocol established with Crassostrea gigas hemocytes previously described.

\section{RESULTS}

\section{Phagocytosis assay}

Microscopical observations of glass-adherent hemocytes from undiluted samples showed a quick internalization of zymosan particles which were observed inside the hemocytes as soon as 5 min after addition. The 2 main types of cells, hyalinocytes and granulocytes of both oyster species, were able to phagocytose yeast walls.

When hemocyte preparations were made from hemolymph collected with MAS (1:1) and subsequently washed in FSW, the phagocytic capacity was similar to that of hemocytes from pure hemolymph.

\section{Chemiluminescence experimental parameter selection with Crassostrea gigas hemocytes}

Effect of MAS concentration on CL. The withdrawal of hemolymph samples, without anti-aggregant MAS, directly into FSW led to immediate high values $\left(2.7 \times 10^{6}\right.$ cpm) of CL activity even without zymosan stimulation. When zymosan stimulation was subsequently applied, a lower peak value $\left(1.8 \times 10^{6} \mathrm{cpm}\right)$ was obtained, i.e. there was a rapid decrease in the signal. For hemolymph samples collected with MAS $(1: 1)$, the CL basis activities of the hemocytes were always very low, about $0.25 \times 10^{6}$ cpm. The CL responses of such hemocytes after stimulation by zymosan varied in intensity according to the MAS concentration (Fig. 1): whereas the CL peak intensities were respectively $1.92 \times 10^{6} \mathrm{cpm}$ and $1.84 \times 10^{6} \mathrm{cpm}$ for $2.5 \%$ and $3.5 \%$ MAS, they were strongly reduced between 4.5 and $10.5 \%$, decreasing from $0.7 \times 10^{6}$ to 0.4 $\times 10^{6} \mathrm{cpm}$. Whatever the MAS concentration, low CL values were observed for the corresponding unstimulated samples (about $0.25 \times 10^{6} \mathrm{cpm}$ ).

Effect of luminol concentration on CL. Comparison of the different luminol-enhanced assays showed a maximal response for $10^{-4} \mathrm{M}$ luminol $\left(3.6 \times 10^{6} \mathrm{cpm}\right.$ with the hemolymph pool assayed) (Fig. 2). At the next higher concentration tested $\left(10^{-3} \mathrm{M}\right)$, the CL peak intensity attained only $0.7 \times 10^{6} \mathrm{cpm}$. At the next lower concentration $\left(10^{-5} \mathrm{M}\right)$, low $\mathrm{CL}$ responses were also observed, $0.8 \times 10^{6} \mathrm{cpm}$. No CL activity was obtained without luminol, nor at the concentrations of $10^{-2} \mathrm{M}$ and $10^{-6} \mathrm{M}$. Therefore, subsequent experiments were all performed at $10^{-4} \mathrm{M}$ final luminol concentration, the equivalent $10^{-3} \mathrm{M} \mathrm{DMSO}$ concentration being without effect on chemiluminescence (data not shown).

Effect of zymosan particle:hemocyte ratio on CL. As previously shown, the contact of hemocytes with zymosan particles led to CL responses. These responses reached a peak generally about 60 min after contact and then decreased slowly over $4 \mathrm{~h}$. However, the level and swiftness of CL response were dependent on the number of zymosan particles added (Fig. 3). A maximum CL activity of $3.84 \times 10^{6} \mathrm{cpm}$ was obtained with a particle:hemocyte ratio of $80: 1$. Higher ratios, 100:1, $120: 1,140: 1$ and $160: 1$, resulted in lower peak values, respectively $1.8 \times 10^{6} \mathrm{cpm}, 2.5 \times 10^{6} \mathrm{cpm}, 1.96 \times 10^{6}$ $\mathrm{cpm}$ and $2.6 \times 10^{6} \mathrm{cpm}$. Lower ratios (from 20:1 to 60:1) resulted in CL responses about $1.5 \times 10^{6} \mathrm{cpm}$.

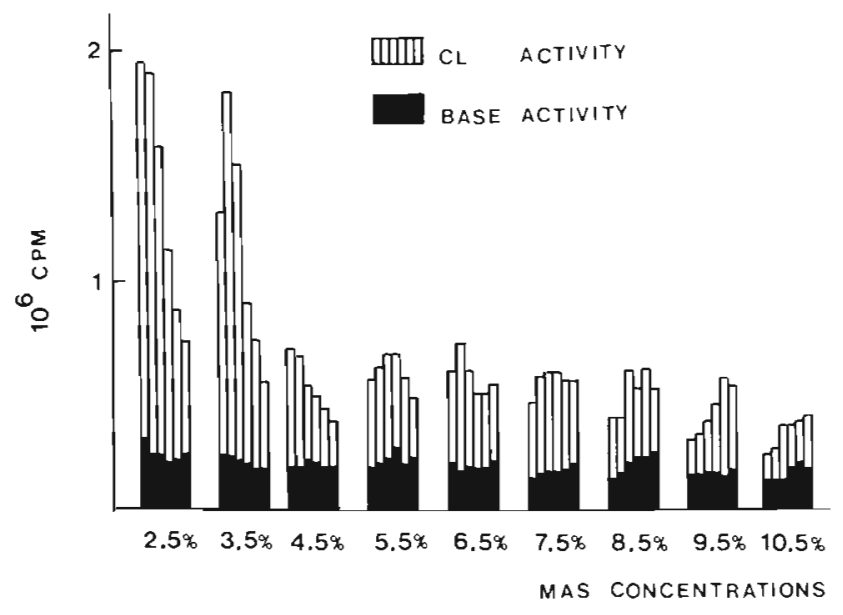

Fig. 1. Chemiluminescence responses of Crassostrea gigas hemocytes (10 oyster pool) for different Modified Alsever Solution (MAS) concentrations in the samples. Particle:hemocyte ratio $80: 1$, luminol concentration $10^{-4} \mathrm{M}$; CL values recorded at 6 time intervals of $26 \mathrm{~min}$ are reported 


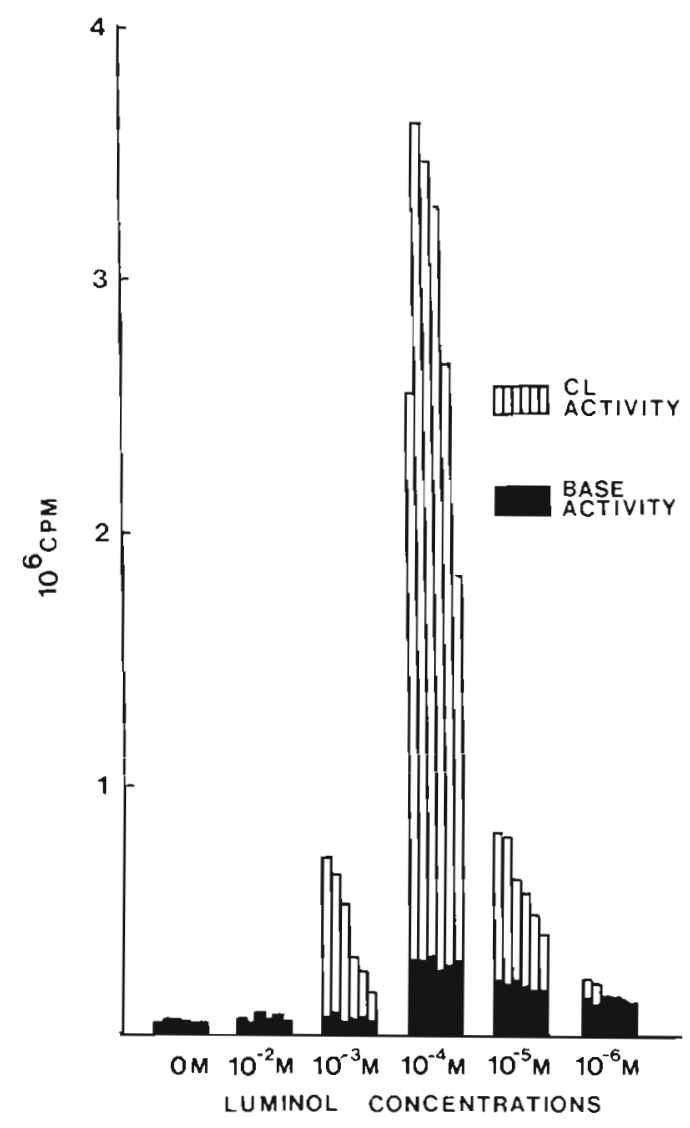

Fig. 2. Effect of luminol concentrations on CL measures of Crassostrea gigas hemocytes, pooled from 10 oysters. Particle: hemocyte ratio 80:1. CL values recorded at 6 time intervals of $26 \mathrm{~min}$ are reported

\section{CL inhibition assays}

Immediately after cytochalasin B treatment, hemocyte preparations showed very strong $\mathrm{CL}$ activity $(3.24$ $\times 10^{6} \mathrm{cpm}$ ) followed by an apparent insensitivity to zymosan stimulation (Fig. 4).

The addition of sodium azide to stimulated hemocytes led to rapid quenching of the CL responses which fell abruptly from $0.92 \times 10^{6} \mathrm{cpm}$ to $0.15 \times 10^{6} \mathrm{cpm}$, whereas at the same time, the stimulated uninhibited control sample reached a peak intensity of $1.10 \times 10^{6} \mathrm{cpm}$.

\section{CL assays of individual hemolymph samples of the two oyster species}

Individual hemolymph samples were analysed for CL responses after zymosan stimulation (particle:hemocyte ratio 80:1) and the number of stimulated hemocyte was constant $\left(2 \times 10^{5}\right)$ for all individuals. Results are presented in Fig. 5 as the CL responses measured at 7 regular time intervals. These values, obtained for 10 specimens of each species, varied considerably in

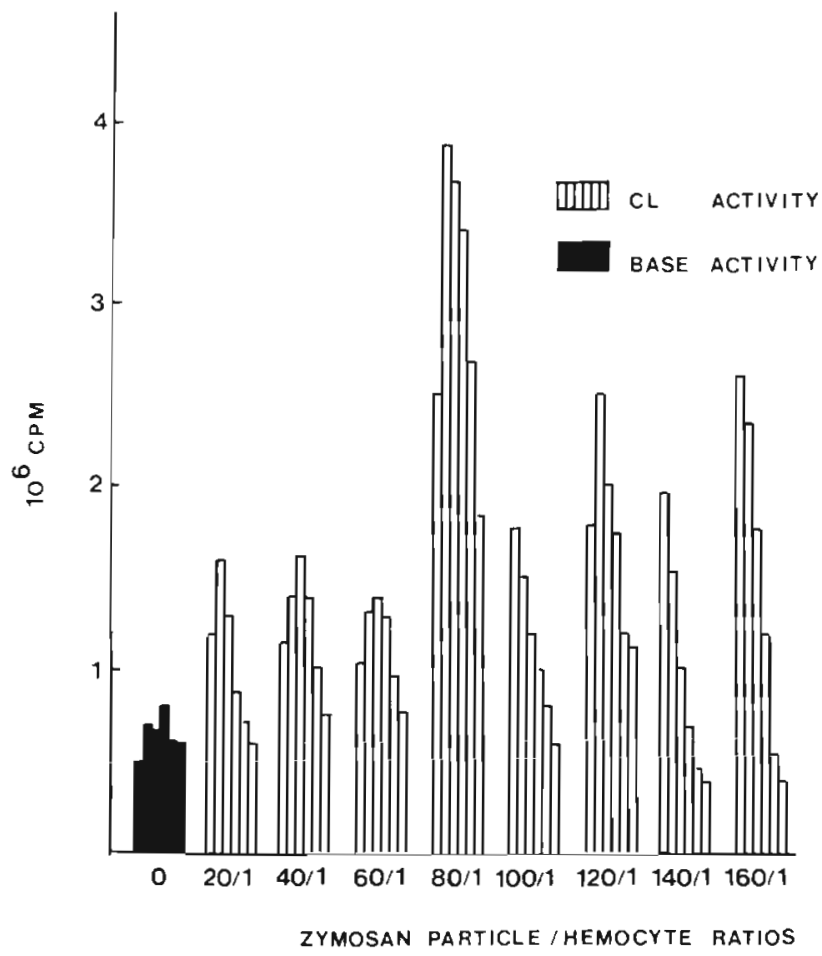

Fig. 3. Chemiluminescence responses of Crassostrea gigas hemocytes (10 oyster pool) stimulated by different zymosan particle:hemocyte ratios. CL values recorded for 6 time intervals of $26 \mathrm{~min}$ are reported. Luminol concentration $10^{-4} \mathrm{M}$

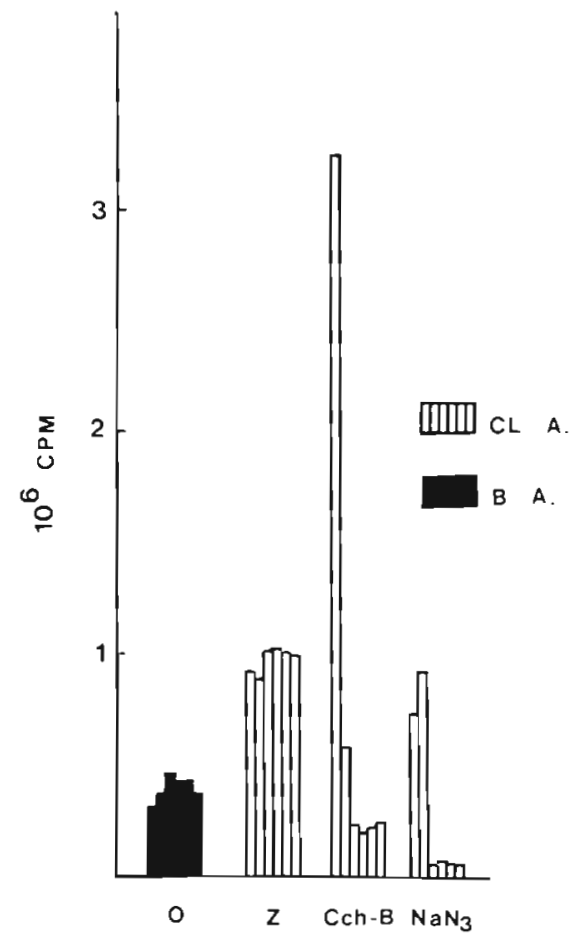

Fig. 4. Effect of cytochalasin B (Cch-B; $\left.1.5 \mu \mathrm{g} \mathrm{ml}^{-1}\right)$ and sodium azide, $\mathrm{NaN}_{3}\left(32 \mathrm{\mu g} \mathrm{m}^{-1}\right)$ on $\mathrm{CL}$ activity of Crassostrea gigas hemocytes (10 oyster pool) stimulated by zymosan $(80: 1)$. Cch $B$ was added just before zymosan; $\mathrm{NaN}_{3}$ was added when the cells reached maximum activity. CL values recorded at 6 time intervals of 26 min are reported 

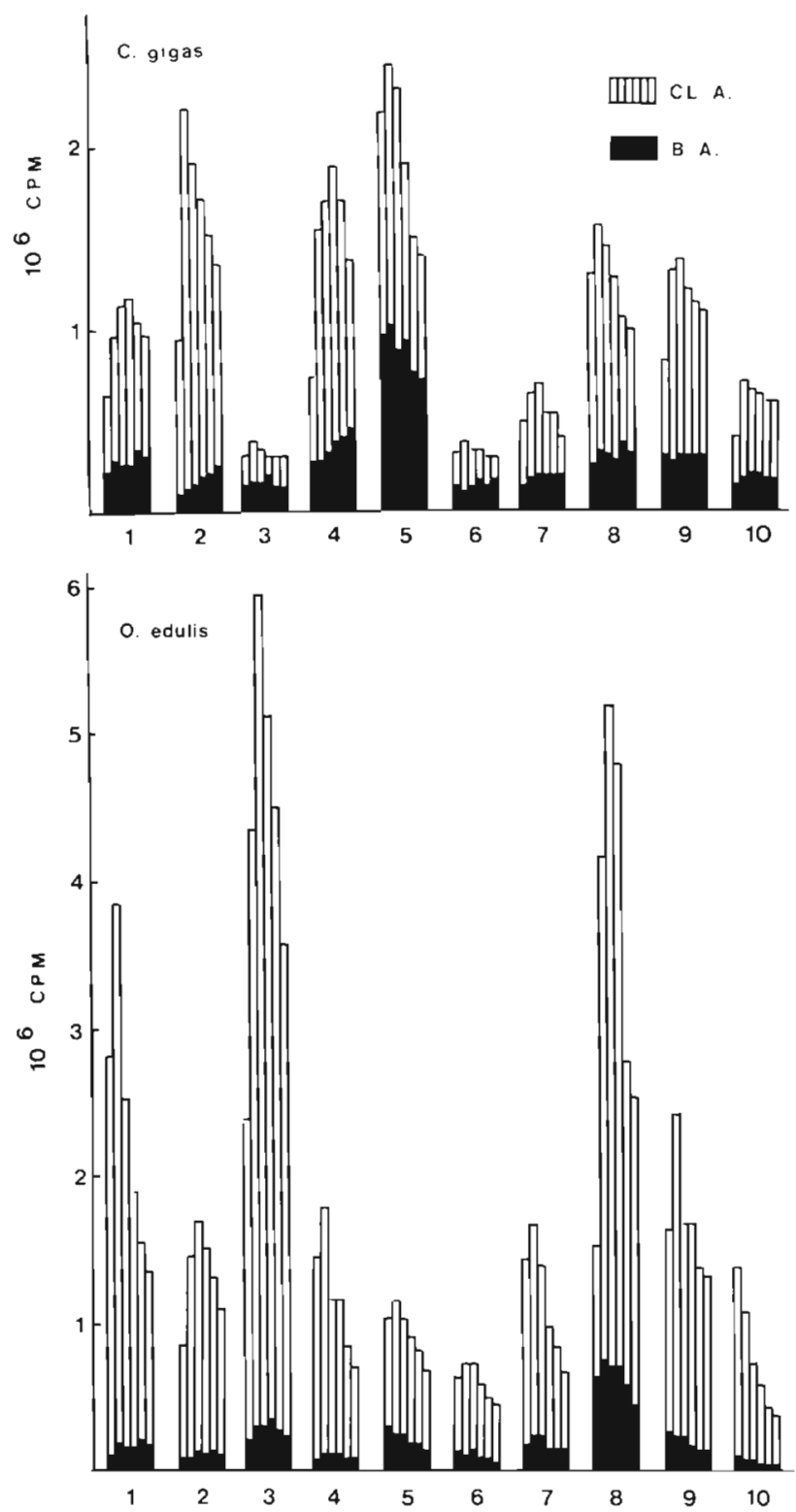

Fig. 5. Chemiluminescence assays on Ostrea edulis and Crassostrea gigas individual hemolymphs (Individual Nos. 1 to 10). Zymosan particle:hemocyte ratio 80:1. CL values recorded at 6 time intervals of $26 \mathrm{~min}$ are reported

intensity and also in swiftness. Taking the peak intensity as a reliable feature (Giltinan et al. 1986) of an individual CL response, comparisons were made between the species. For Ostrea edulis, CL values ranged about a mean of $2.59 \times 10^{6} \mathrm{cpm}\left( \pm 1.6 \times 10^{6}\right.$ $\mathrm{cpm})$ and for Crassostrea gigas about $1.28 \times 10^{5} \mathrm{cpm}$ $\left( \pm 0.63 \times 10^{6} \mathrm{cpm}\right.$ ) (Table 1). The individual and species CL variability was statistically analysed. Using a correlation analysis, it was shown for both oyster species that the CL activity of the hemocytes was independent of the quantity of MAS present in the samples $(\mathrm{r}=0.15$ for $O$. edulis and $\mathrm{r}=0.62$ for $C$. gigas; $\mathrm{df}=8$, for $\mathrm{r}=0.63$, the probability is $\mathrm{p}=0.05$ ).
Since individual variability was established between hemolymph samples with strictly similar hemocyte numbers, the relation between CL peak intensity and the percentage of the different cell types was tested by the estimation of the correlation coefficients $r$. The results, presented in Table 1, indicate a statistically significant relation between CL response and percentage of granulocytes, and also between CL response and percentage of small hyalinocytes, for both oyster species. Nevertheless, the CL response of Crassostreas gigas was high when the percentage of small hyalinocytes $(\mathrm{SH})$ was low $(\mathrm{r}=-0.73)$ and when the proportion of granulocytes (G) was high ( $\mathrm{r}=0.75$ ). In Ostrea edulis, a contrary relation was observed ( $\mathrm{r}=$ 0.64 for the relation between CL response and \% $\mathrm{SH}$, and $\mathrm{I}=-0.64$ for the relation between $\mathrm{CL}$ response and $\%$ G). No correlation was found between the CL activity of the oyster hemolymph and the quantity of large hyalinocytes $(r<$ theoretical $r$ ).

\section{DISCUSSION}

The chief aim of this work was to establish experimental parameters in order to reliably apply the CL technique to the study of phagocytic function and respiratory burst activity of the hemocytes of 2 oyster species, Ostrea edulis and Crassostrea gigas. This technique was selected from the numerous methods of phagocytosis measurement because CL is considered a valuable and sensitive tool for monitoring the potential activity of phagocyte populations in vertebrates and, therefore, the immunological state of an animal. So we chose CL as the method for quantification of defense responses of individual oysters, looking for a correlation between hemogram features and hemocyte activities

As also demonstrated in several gastropod species (Dikkeboom et al. 1988b), we showed by the CL technique that oyster hemocytes display metabolic events associated with the phagocytosis of zymosan particles. In practice, the study was not a simple extrapolation of gastropod studies, since the establishment of the CL technique required the definition of several controlled experimental conditions, which had to be adapted to oyster hemocytes.

The first problem encountered with bivalve hemocytes is the rapid aggregation and clumping of the cells. As previously described (Bachère et al. 1988), this difficulty may be greatly reduced by instantaneously diluting the hemolymph, during withdrawal, in MAS, an efficient anti-aggregant. In this way, it is subsequently possible to aliquot hemolymph into several samples which are strictly homogeneous, quantitatively and qualitatively, and consequently well- 
Table 1. Ostrea edulis and Crassostrea gigas. Correlation between chemiluminescence responses of 10 individual oyster hemolymphs, MAS concentrations and hemogram characteristic (cell type percentages) in the respective samples. The peak values obtained for each individual assay, considered as representative of CL activities, were used in correlation tests. G: granulocyte; LH: large hyalinocyte; SH: small hyalinocyte

\begin{tabular}{|c|c|c|c|c|c|c|c|c|c|c|}
\hline \multirow[t]{3}{*}{ Ind. no. } & \multicolumn{5}{|c|}{ O. edulis } & \multicolumn{5}{|c|}{ C. gigas } \\
\hline & \multirow{2}{*}{$\begin{array}{c}C L \\
\text { response } \\
\left(10^{6} \mathrm{cpm}\right)\end{array}$} & \multirow{2}{*}{$\begin{array}{c}\text { MAS conc. } \\
(\%)\end{array}$} & \multicolumn{3}{|c|}{ Cell types $(\%)$} & \multirow{2}{*}{$\begin{array}{c}C L \\
\text { response } \\
\left(10^{6} \mathrm{cpm}\right)\end{array}$} & \multirow{2}{*}{$\begin{array}{c}\text { MAS conc. } \\
(\%)\end{array}$} & \multicolumn{3}{|c|}{ Cell types $(\%)$} \\
\hline & & & G & LH & $\mathrm{SH}$ & & & G & $\mathrm{LH}$ & SH \\
\hline 1 & 3.84 & 3.1 & 7 & 21 & 72 & 1.18 & 2.4 & 3 & 85 & 12 \\
\hline 2 & 1.70 & 2.5 & 23 & 38 & 39 & 2.2 & 2.8 & 20 & 62 & 18 \\
\hline 3 & 5.95 & 1.1 & 9 & 29 & 62 & 0.37 & 1.6 & 4 & 69 & 27 \\
\hline 4 & 1.80 & 3.1 & 37 & 30 & 33 & 1.87 & 2.0 & 8 & 79 & 13 \\
\hline 5 & 1.17 & 1.9 & 29 & 48 & 23 & 2.47 & 1.6 & 6 & 79 & 15 \\
\hline 6 & 0.75 & 2.1 & 42 & 34 & 24 & 0.38 & 1.2 & 5 & 67 & 28 \\
\hline 7 & 1.70 & 1.4 & 26 & 48 & 26 & 0.69 & 1.8 & 1 & 72 & 27 \\
\hline 8 & 5.20 & 3.1 & 27 & 49 & 24 & 1.56 & 2.4 & 6 & 77 & 17 \\
\hline 9 & 2.45 & 1.6 & 47 & 31 & 22 & 1.38 & 3.1 & 4 & 79 & 17 \\
\hline \multirow[t]{2}{*}{10} & 1.40 & 1.8 & 57 & 28 & 15 & 0.69 & 1.8 & 3 & 75 & 22 \\
\hline & $\begin{array}{c}\text { Mean }= \\
2.59 \\
\pm 1.8\end{array}$ & $\begin{array}{l}r= \\
0.15\end{array}$ & $\begin{array}{c}r- \\
-0.64\end{array}$ & $\begin{array}{l}r= \\
0.07\end{array}$ & $\begin{array}{c}r= \\
0.64\end{array}$ & $\begin{array}{c}\text { Mean }= \\
1.28 \\
\pm 0.7\end{array}$ & $\begin{array}{l}r= \\
0.62\end{array}$ & $\begin{array}{c}r= \\
0.75\end{array}$ & $\begin{array}{c}r= \\
0.05\end{array}$ & $\begin{array}{c}r= \\
-0.73\end{array}$ \\
\hline
\end{tabular}

adapted for performing statistically comparable assays This methodology also had the advantage of allowing the cells to be kept in a non-stimulated state. In contrast, hemolymph collected without MAS, or in FSW led to a high spontaneous CL activity of hemocytes which interfered with their subsequent phagocytic stimulation by zymosan. This CL activity, apparently independent of external stimulation, suggests an autoactivation phenomenon. Contact with the syringe wall or between hemocytes may be sufficient to activate the cells because it leads to perturbation of the hemocyte membrane and probably to depolarization, setting off metabolic events and CL (Van Dyke et al. 1981). This depolarization is dependent on transmembrane movements of $\mathrm{Ca}^{2+}$ and $\mathrm{Na}^{+}$ions and is prevented by the chelator EDTA, as shown for some vertebrate blood cells which can be kept in a quiescent state in medium without $\mathrm{Ca}^{2+}$ until the start of CL assays. However, the phagocytic process needs a sufficient quantity of $\mathrm{Ca}^{2+}$ and $\mathrm{Mg}^{2+}$, which must be supplied by the assay medium (Trush et al. 1978, Allen 1986). MAS appeared particularly efficient for oyster hemocytes because it permits passage from a quiescent non-aggregated state to a stimulated sensitive state. This stage change, proved by histological examination of phagocytosis recovery when FSW was added to MAS-diluted hemocyte suspension, was also established by CL provided that the final MAS concentration was about $2.5 \%$ but did not exceed $3.5 \%$. Thus, MAS/hemolymph (1:1) mixtures need to be diluted 20-fold in FSW to give $2.5 \%$ MAS. Taking into account the range in the number of hemocytes ( 1 to $6 \times 10^{6}$ hemocytes $\left.\mathrm{ml}^{-1}\right)$ and the hemolymph sample size (about $0.5 \mathrm{ml}$ ), several $2 \mathrm{ml}$ aliquots $\left(1\right.$ to $3 \times 10^{5}$ hemocytes aliquot $\left.{ }^{-1}\right)$ can be obtained from a hemolymph sample. Given that small differences in MAS concentrations (around $2.5 \%$ ) did not affect $C L$, the hemocyte number could be strictly fixed, leading to a comparative aliquoted sample series. In practice, $2 \times 10^{5}$ hemocytes per aliquot proved to be adequate to measure a sufficient quantity of $\mathrm{CL}$ during a $0.5 \mathrm{~min}$ counting period, the assays being performed using luminol as signal enhancer. Indeed, a primary advantage of luminol is to decrease the number of cells required to obtain a significant signal (Allen \& Loose 1976). The $10^{-4} \mathrm{M}$ luminol concentration gave the optimal amplification of the $C L$ response whereas at higher concentrations CL responses were reduced or absent, possibly resulting from a toxic effect of the luminol and/or of the DMSO solvent on the hemocytes.

After these experimental parameters had been established, the CL response itself was defined by testing inhibitors of known levels of phagocytic activity. The strong inhibition of CL response by cytochala$\sin \mathrm{B}$ indicated that $\mathrm{CL}$ phenomena were linked to phagocytosis activation of the hemocytes since cytochalasin B interferes with the cytoskeletal movements of the cells. The sudden flash of light observed when adding cytochalasin B may be related to extracellular release of lysosomal enzymes and generation of superoxide ions, $\mathrm{O}_{2}{ }^{-}$(Hoffstein et al. 1982). Subsequent contact with zymosan particles, which cannot 
be internalized, did not lead to activation of respiratory burst.

When added at CL peak activity, sodium azide had an immediate and strong inhibitory effect on the luminol-enhanced system. Since sodium azide is both a quencher of singlet oxygen ${ }^{1} \mathrm{O}_{2}$ and a peroxidase inhibitor, and because luminol, as a chemiluminescent bystander molecule, may be an indicator of the myeloperoxidase- $\mathrm{H}_{2} \mathrm{O}_{2}$-halide system (De Chatelet et al. 1982), it may be inferred that the respiratory burst in bivalves resembles the mechanism in vertebrate phagocytosis. To confirm this hypothesis further research on the biochemical processes of $\mathrm{O}_{2}$-dependent killing activity in bivalve hemocytes is needed.

As reported for CL assays on the snail Lymnaea stagnalis (Dikkeboom et al. 1987), we have shown stimulus-ratio-dependent responses for oyster hemocytes. An optimal zymosan:hemocyte ratio of 80:1 was selected and used in all further experiments. The variable $C L$ responses obtained with the different pooled hemolymph samples during the standardization phase suggested an inter-and intra-specific variability of CL response to the same stimulus. This was confirmed in assays with hemolymph samples from individual oysters $(n=10)$ containing the same number of hemocytes. The CL responses of Ostrea edulis ranged around $2.35 \times 10^{6} \mathrm{cpm}\left( \pm 1.67 \times 10^{6} \mathrm{cpm}\right)$, whereas for Crassostrea gigas, cell activity was $0.97 \times 10^{6} \mathrm{cpm}$ $\left( \pm 0.63 \times 10^{6}\right) \mathrm{cpm}$. Before conclusions can be made about the significance of these observations, further analyses will be necessary, particularly with oysters collected from different areas and at different times. The reliability of the CL protocol now makes such research possible.

The intraspecific individual CL variability was caused by variability in the granulocyte:small hyalinocyte ratio, although this correlation differed between the 2 oysters. In order to understand the influence of hemogram characteristics on CL responses, the respective involvement of each cell type in CL activity can best be studied with pure hemocyte populations separated using isopycnic and elutriation centrifugation (Bachère et al. 1988).

In the present poor state of knowledge on molluscan defense mechanisms, the CL technique could be particularly suitable for analysing the immunological state of oysters. In studying immune responses against pathogens or pollutants (Wishkovsky 1988), it will be possible to investigate stressor-hemocyte interactions in vitro, to follow defense activity in time for individual oysters and to gain understanding of the development or remission of pathological processes. From a more applied point of view, CL could also be considered as an interesting marker to test for individual genetic selection. With the recent development of hemocyte separation protocols
(Bachère et al. 1988) and of native pathogen purification (Mialhe et al. 1988), such a technique appeared necessary for investigating the killing mechanisms of bivalves hemocytes, and for comparative studies on antimicrobial defense capacity between individuals. Moreover, from a parasitological point of view, research could be undertaken on the interactions of intrahemocytic parasites such as Bonamia ostreae with their hosts, in order to understand and to compare their survival mechanisms with those of vertebrate macrophage parasites such as Leishmania (Alexander \& Russel 1985), Toxoplasma and Trypanosoma (Moulder 1985, Zingales \& Colli 1985).

Acknowledgements. We are grateful to Dr W. P. W. Van Der Knaap for helpful criticism of the manuscript.

\section{LITERA.TURE CITED}

Alexander, J., Russel, D. G. (1985). Parasite antigens, their role in protection, diagnosis and escape: the leishmanioses. Curr. Topics Microbiol. Immunol. 120: 43-67

Allen, R. (1986). Phagocytic leukocyte oxygenation activities and chemiluminescence: a kinetic approach to analysis. In: DeLuca, M. A., McElroy, W. D. (eds.) Meth. Enzym. 133. Academic Press, New York, p. 449-493

Allen, R. C., Stjernholm, R. L., Steele, R. H. (1972). Evidence for the generation of an electronic excitation state in human polymorphonuclear leukocytes and its participation in bactericidal activity. Biochem. Biophys. Res. Commun. 47:679

Allen, R. C. Loose, L. D. (1976). Phagocytic activation of a luminol-dependent chemiluminescence in rabbit alveolar and peritoneal macrophages. Biochem. Biophys. Res. Commun. 69: 245

Andrew, P. W., Jackett, P.S., Lowrie, D. B. (1985). Killing and degradation of microorganisms by macrophages. In: Dean, R. T., Jemp, W. (eds.) Mononuclear phagocytes physiology and pathology. Elsevier, Amsterdam, p. 311-336

Bachère, E. , Chagot, D. Grizel, H. (1988). Sepąration of Crassostrea gigas hemocytes by density gradient centrifugation and counterflow centrifugal elutriation. Dev. comp. Immunol. 12: 546-559

Badwey, J. A., Karnosky, M. L. (1980). Active oxygen species and the functions of phagocytic leukocytes. Ann. Rev. Biochem. 49: 695-726

Bang, F. B. (1975). Phagocytosis in invertebrates. In: Maramoroschi, K., Hope, R. E. (eds.) Invertebrate immunity. Academic Press, New York, p. 137-151

De Chatelet, L. R., Long, G. D., Shirley, P. S., Bass, D. A. Thomas, M. J., Henderson, F. W., Cohen, M. S. (1982). Mechanism of the luminol-dependent chemiluminescence of human neutrophils. J. Immunol. 129: 1589-1593

Dikkeboom, R., Tijnagel, J. M. Mulder, E., Van Der Knaap. W. P. W. (1987). Hemocytes of the pond snail Lymnaea stagnalis generate reactive forms of oxygen. J. Invert. Pathol. 49: 321-331

Dikkeboom, R., Bayne, C. J., Van Der Knaap. W. P. W., Tijnajel, J. M. G. H. (1988a). Possible role of reactive forms of oxygen in vitro killing Schistosoma mansoni sporocysts by hemocytes of Lymnaea stagnalis. Parasitol. Res. 75: $148-154$ 
Dikkeboom, R., Van Der Knaap, W. P. W., Van Den Bovenkamp, W., Tijnagel, J. M., Bayne, C. J. (1988b). The production of toxic oxygen metabolites by hemocytes of different snail species. Dev. comp. Immunol. 12: 509-520

Fromtling, R. A.. Abruzzo, G. K. (1985). Chemiluminescence as a tool for the evaluation of antimicrobial agents: a review. Meth. Find. expt. Clin. Pharmacol. 7: 493--500

Giltinan, D. M., Capizzi, T P., Abroszzo, G. K., Fromtling, R. A. (1986). Design and analysis consideration in evaluating the chemiluminescence response of mouse spleen cells. J. Clin. Microb. 23: 531-535

Halliwell, B., Guterridge, J. M. C. (1984). Oxygen toxicity, oxygen radicals, transition metals and disease. Biochem. J. 219: 1-14

Herzenberg, L. A. (1979). Antigen specific identification and cloning of hybridomas with a fluorescence activated cell sorter (FACS). Proc. natn. Acad. Sci. 76: 1962

Hoffstein, S. T., Korchak, H. M., Smolen, J. E., Weissmann, G. (1982). Early consequences of neutrophil activation and their association with degranulation. In: Kornowsky, M. L., Bolis, L. (eds.) Phagocytosis - past and future. Academic Press, New York, p. 47-65

Mialhe, E., Bachère, E., Chagot, D., Grzel, H. (1988). Isolation and purification of the protozoan Bonamia ostreae (Pichot et al. 1979), a parasite affecting the flat oyster Ostrea edulis L. Aquaculture 71: 293-289

Responsible Subject Editor: A. K. Sparks, Seattle, Washington, USA
Moulder, J. W. (1985). Comparative biology of intracellular parasitism. Microbiol. Rev. 49: 298-337

Nakamura, M. Mori, K. Innoka, S., Nomura, T (1985). In vitro production of hydrogen peroxide by the amoebocytes of the scallop, Patinopecten yessoensis (JAY). Dev. comp. Immunol. 9: 407-417

Spitznagel, K. (1984). Nonoxidative antimicrobial reactions of leukocytes. Contemp. Topics Immunobiol. 14: 283-343

Thomas, E. L., Lehrer, R. I., Rest, R. F. (1988). Human activity neutrophil antimicrobial activity. Rev. infect. Dis. 10: $450-456$

Trush, M. A., Wilson, M. E., Van Dyke, K. (1978). The generation of chemiluminescence (CL) by phagocytic cells. Meth. Enzymol. 8: 462-494

Van Dyke, K., Van Dyke, C., Peden, D., Matomoros, M., Castranova, V., Jones, G. (1981). Preliminary events leading human granulacytes. In: De Luca, M., Mc Elroy, W. (eds.) Bioluminescence and chemiluminescence. Academic Press, New York, p. 45-53

Wishkovsky, A. (1988). Chemiluminescence: an advanced tool for measuring phagocytosis. In: Fisher, W. S. (ed.) Disease processes in marine bivalve molluscs. American Fisheries Society Special Publication 18, p. 292-298

Zingales, B., Colli, W. (1985). Trypanosoma cruzi: interaction with host-cells. Curr. Topics Microbiol. Immunol. 117: $123-152$

Manuscript first received: December 4, 1990

Revised version accepted: July 8, 1991 